\title{
Using Capacitance Sensor to Extract Characteristic Signals of Dozing from Skin Surface
}

\author{
Po-Ying Chen, ${ }^{1}$ Chi-Chang Chen, ${ }^{2}$ Wen-Kuan Yeh, ${ }^{3}$ Yukan Chang, ${ }^{2}$ \\ Der-Chen Huang, ${ }^{4}$ Shyr-Shen Yu, ${ }^{4}$ Chwei-Shyong Tsai, ${ }^{5}$ Yu-Jung Huang, ${ }^{6}$ Wei-Cheng Lin, ${ }^{7}$ \\ Shao-I Chu, ${ }^{8}$ Chung-Long Pan, ${ }^{7}$ Tsung-Hung Lin, ${ }^{9}$ and Shyh-Chang Liu ${ }^{2}$ \\ ${ }^{1}$ Department of Electronic Engineering, National Chin-Yi University of Technology, Taichung 41170, Taiwan \\ ${ }^{2}$ Department of Information Engineering, I-Shou University, Kaohsiung 84001, Taiwan \\ ${ }^{3}$ Department of Electronic Engineering, National Kaohsiung University, Kaohsiung 811, Taiwan \\ ${ }^{4}$ Department of Computer Science and Engineering, National Chung Hsing University, Taichung 402, Taiwan \\ ${ }^{5}$ Department of Management of Information System, National Chung Hsing University, Taichung 402, Taiwan \\ ${ }^{6}$ Department of Electronic Engineering, I-Shou University, Kaohsiung 84001, Taiwan \\ ${ }^{7}$ Department of Electrical Engineering, I-Shou University, Kaohsiung 84001, Taiwan \\ ${ }^{8}$ Department of Electronic Engineering, National Kaohsiung University of Applied Sciences, Kaohsiung 811, Taiwan \\ ${ }^{9}$ Department of Computer Science and Information Technology, National Chin-Yi University of Technology, Taichung 41170, Taiwan
}

Correspondence should be addressed to Chi-Chang Chen; ccchen@isu.edu.tw

Received 21 May 2014; Revised 20 October 2014; Accepted 23 October 2014; Published 13 November 2014

Academic Editor: Chengkuo Lee

Copyright (c) 2014 Po-Ying Chen et al. This is an open access article distributed under the Creative Commons Attribution License, which permits unrestricted use, distribution, and reproduction in any medium, provided the original work is properly cited.

\begin{abstract}
Skin is the largest organ of the human body and a physiological structure that is directly exposed to the environment. From a theoretical perspective, numerous physiological and psychological signals use the skin as a medium for input and output with the outside world. Therefore, the skin is considered an optimal signal interception point when developing noninvasive, direct, and rapid signal exploration devices. To date, skin signal interceptions are predominantly performed by measuring skin impedance. However, this method is prone to interference such as sweat secretion, salt accumulation on the skin, and muscle contractions, which may result in a substantial amount of interference and erroneous results. The present study proposes novel and effective methods for skin signal interception, such as using a nested probe as a sensor to measure capacitance to be further processed as physiological and psychological signals. The experimental results indicate that the capacitance curve for the transition between wakefulness and dozing exhibits significant changes. This change in the curve can be analyzed by computer programs to clearly and rapidly determine whether the subject has entered the initial phases of sleep.
\end{abstract}

\section{Introduction}

A physiological exploration platform that is (1) easily constructed, (2) easily operated, (3) programmable, (4) capable of producing amplifiable and extractable signals, and (5) noninvasive can be effectively achieved by measuring characteristics of the skin surface while applying electricity on it [1-3]. Depending on how electricity is applied, these characteristics theoretically can be measured using capacitance or electrical bioimpedance amplifiers. Unlike previous studies, which have focused mainly on measuring impedance, the present study measures both signals. Two more characteristics of skin signals are discovered in this study. Under low voltage bias, the measured signals do not change over time but differ from person to person and thus can be used for personal identification and information safety. Under high voltage bias, on the other hand, the measured signals vary with physiological and psychological status and thus can be used for physiological signal diagnosis and remote medical applications. It is this second characteristic of skin signals that were used in this study to detect dozing. 


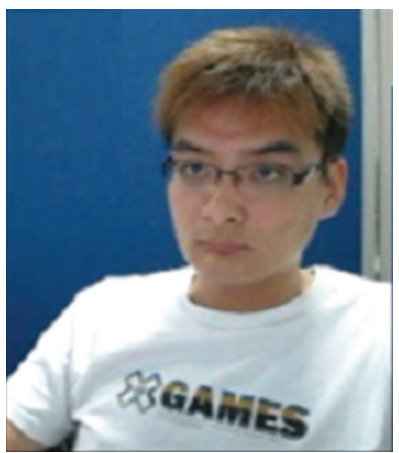

(a)

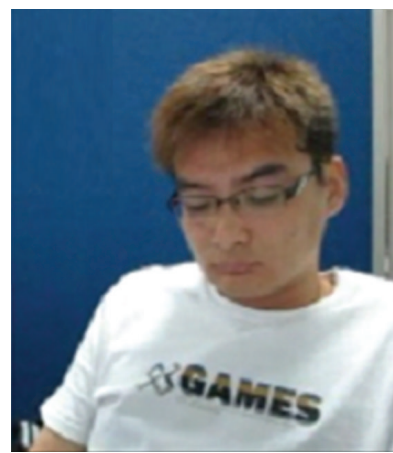

(b)

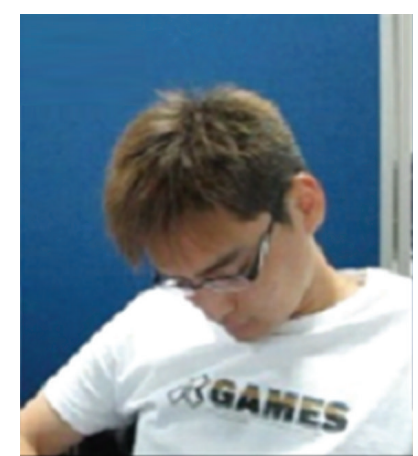

(c)

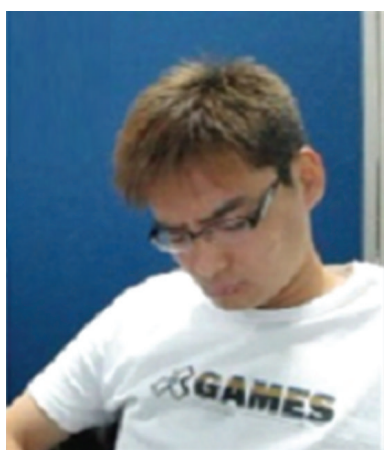

(d)

FIgURE 1: The four stages of dozing.

The surface of the skin is characterized by being conductive, and signals can change rapidly. Changes in electric signals are closely related to psychological processes. Research has been conducted on skin signals over the past fifty years and is increasingly widespread. In recent years, studies have demonstrated that electrodermal activity (EDA) and skin conductance are correlated with changes in the sweat glands and sympathetic branch activities of the autonomic nerve system (ANS).

Measuring EDA has become an essential method for examining the psychological process of sympathetic excitement in research on emotions because the ANS is extremely effective in inducing emotions and motivation. Direct methods for evaluating neural activity have been developed in recent years (e.g., positron emission tomography) [3-5]. Skin conductance is reflected by neural and cerebral activity; consequently, relevant applying techniques for measuring EDA are relatively inexpensive. In addition, numerous studies have focused on these techniques because they are active and relatively reliable. Analyzing skin conductance is ideal for researching the process of psychological or emotional changes because the measuring processes can be performed continually without causing discomfort to the subject. In addition, skin conductance constitutes a feasible method for providing an indicator of changes in feelings and emotions in subjects while making judgments [6-16].

Numerous studies have focused on exploring skin signals, the majority of which have used skin impedance or skin conductance to intercept signals. However, skin impedance can be influenced by many factors, such as sweat secretion, salt accumulation, and muscle contractions. These factors affect skin conductance, which subsequently causes noise and leads to erroneous results. Furthermore, this measurement method is inaccurate during dynamic exercises.

The present study proposes using an alternative method to measure skin signals, which involves using a nested probe as a sensor to measure capacitance to be further processed as physiological and psychological signals. The measurement design of the present study was developed based on the fact that the stratum corneum is thin and exhibits excellent insulation. Mookerjea et al. has proven that capacitive characteristics are crucial when the insulation is thin [17]. Therefore, the metal-insulator-metal (MIM) structure of capacitance is ideal for detecting skin signals. The structure of human skin accounts for two-thirds of the required MIM structure; thus, signals within the body can be detected by simply using an additional metal array probe. Fundamental theories of the MIM capacitance structure have been well established through scientific and technological development in the past few decades. Therefore, MIM capacitance is an excellent method for explaining skin signals. The focus of current studies has been to explore research topics related to this method further; thus, the present study used skin capacitance to measure data and subsequently extract the characteristic signals of dozing.

Dozing is a near-sleep physiological state, which all humans have experienced. Common behaviors that occur during dozing include (1) blurry vision and swollen eyes, (2) body movements such as nodding, (3) repeated yawns, (4) reduced focus that causes judgment errors, (5) slow or abnormal reactions, (6) reduced attention and impaired thinking abilities, and (7) slow movements and the loss of a sense of direction $[9,10]$. A substantial number of relevant studies have addressed the topic of drowsy driving. The present study explores the continual process of alternation between wakefulness (Stage W) and light sleep (Stage 1) during the sleep cycle, which is referred to as the process of dozing. The average human being experiences unpredicted dozing for a short period of two to three minutes. Figure 1 shows the four stages of dozing. Somnolent behavior is illustrated from left to right in the figure. When human beings experience this observable mental struggle, the physiological environment of the human body, specifically the capacitance signal, is shown in this study to exhibit characteristics that greatly differ from that of the body in a wakeful state.

\section{Experimental Details}

2.1. Subjects. A total of 38 men and 2 women between the ages of 20 and 30 were recruited as the experimental subjects. The sampling period for the skin capacitance measurement was $0.005 \mathrm{sec}$, corresponding to a sampling frequency of $200 \mathrm{~Hz}$. The measurements were performed between 10:00 and 11:00 p.m., and each measurement unit was 5 minutes in length. 

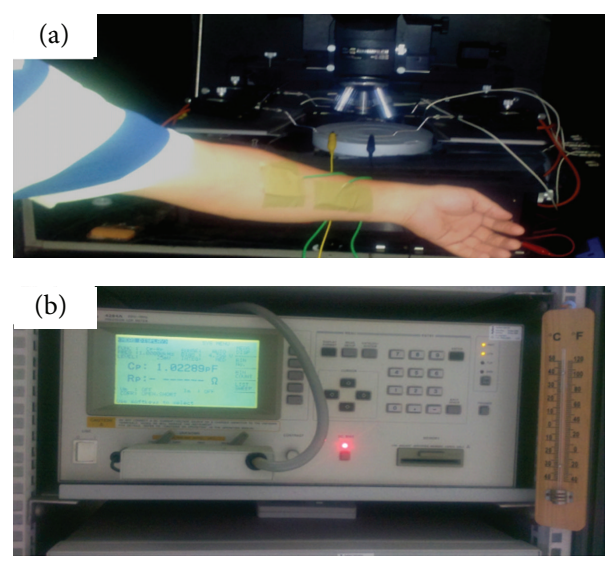

FIGURE 2: Experimental equipment.

The subjects were tested after a full day of normal routine without sleeping during work hours. Intense exercise and other stimulating behaviors were prohibited one hour before the experiment. An electrical capacity measurement device was attached to the subjects throughout the experiment.

Points in time when the subjects experienced somnolent behavior were documented, and data packets during the occurrence of somnolent behavior were analyzed. Two minutes of physiological data for each of the three stages (Stage W, dozing, and Stage 1) were observed and the last 128 measurements were extracted for further analysis.

2.2. Experimental Hardware. An Agilent 4284A Precision LCR Meter was employed. The meter covers a frequency range between $5 \mathrm{~Hz}$ and $3 \mathrm{GHz}$ and includes a programmable wireless DC bias sweep source that can be applied in the fields of physiology and medicine. Measurements were conducted by attaching a grid metal mesh to the skin at the point of measurement (Figure 2), thereby directly exporting the capacitance measurement results to an Excel spreadsheet.

Before analyzing and discussing the experimental details, basic terms should first be defined. In circuitry, capacitance (symbolized as C) is defined as the ability of a body to store an electrical charge under a given voltage. The unit for capacitance is farads (symbolized as F), which is included in the International System of Units. A parallel plate capacitor is a simplistic device that comprises two parallel conductor plates that are separated by space or dielectrics. Assuming that the two plates are, respectively, loaded with negative and positive charges, the respective charges for the two plates are $-Q$ and $+Q$, and the electrical potential difference between the two plates is $+V, C=Q / V$. Capacitance is in directly proportional and inversely proportional to the area of the plate and the distance between the plates, respectively. Assuming that the distance between the plates, $d$, is substantially smaller than that of the length and width of the plates, the previously mentioned equation is of good fit. The electric field is evenly distributed throughout the area. The fringe electric field surrounding the capacitor provides only a minimal contribution; thus, it can be disregarded.

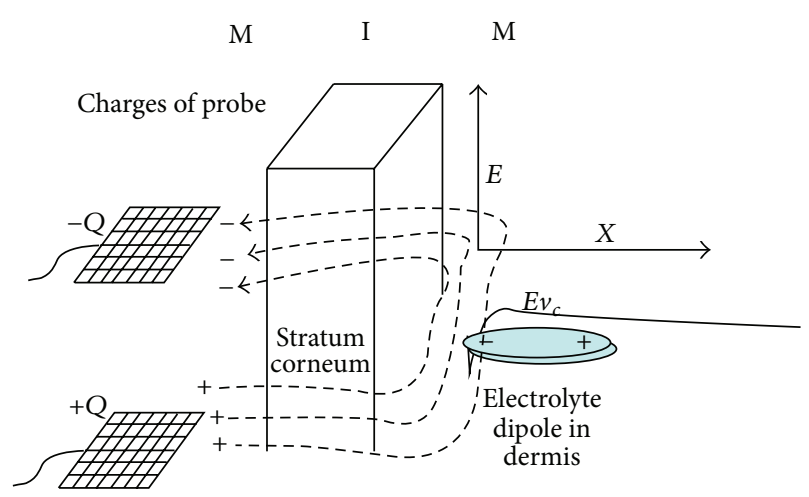

FIGURE 3: The energy band used for skin signal measurement.

\section{Results and Discussion}

3.1. Discussion of the Results. Figure 3 shows the energy band diagram obtained using a MIM structure with bias when conducting skin capacitance measurements. When using bias to scan and measure the circuit-voltage $(C-V)$ of the skin surface, an additional mesh electrode was used as a conductor (M) and the stratum corneum was used as an insulator (I). The dermis tissue can be considered as an additional conductor $(\mathrm{M})$. Lines of electric force penetrated the insulated layer at the center of the MIM structure and formed an electric field $(E)$. Under ideal circumstances (i.e., when the area of the measurement conductor is sufficiently large) the measured $C$ is equal to $E / 2 \varepsilon_{0}$, where $\varepsilon_{0}$ represents the ideal dielectric constant. In other words, the skin capacitance measurements were determined by the electric field $(E)$.

The energy band on the left in Figure 3 represents the energy profile sent out through the attached mesh electrode. The shape of this energy profile is determined based on the number and quality of electric charges on the mesh electrode, where electric charge quality refers to the distribution of electric charges. As a result of MIM interaction, this energy profile causes the dermis conductor to develop a corresponding energy profile of its own, which is shown as an energy band on the right in Figure 3. The correspondence, however, is complicated by the presence of various sophisticated systems in the human body such as the nerve system.

In the human body, electric charge distribution is presented by the dipoles of the charged electrolytes in the tissue fluid. All signals in the human body are constantly and naturally presented through the arrangement of electric dipoles. These signals are difficult to measure directly, but they are coupled with known electrical charges on the meshed electrodes to form the measured overall signals of the skin capacitance. A certain number of electric dipoles are flipped and rearranged after sensing the distribution of positive and negative charges on the mesh electrodes. Flipping occurs during $C-V$ measurements with external bias. However, flipping requires lag time (Tc) because the charged electrolyte dipoles possess volume and mass. Thus, crucial signals can be restored by scanning the $C-V$ characteristic figures with high and low frequencies. 


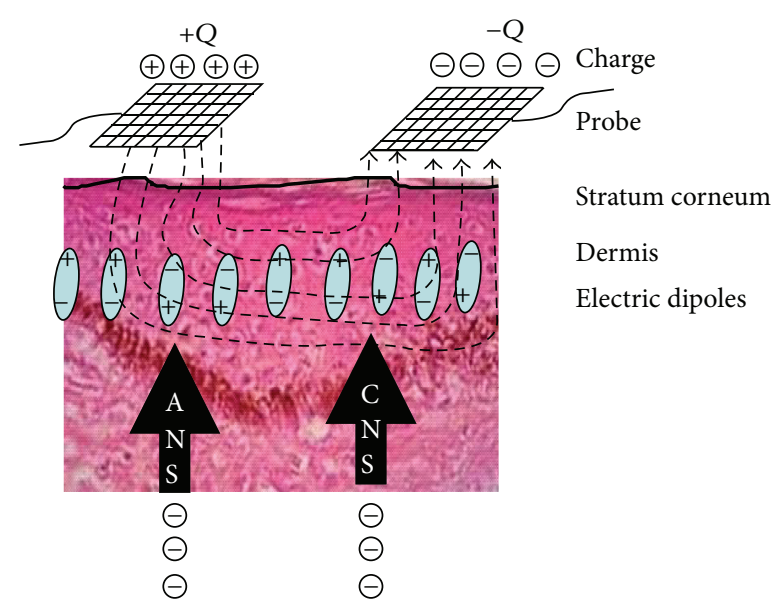

FIGURE 4: Experimental model of this paper.

In this work, the experimental model is the use of capacitive measuring device to probe for physiological signals affected by electrolyte electric dipole array beneath the skin stratum corneum. The capacitance MIM (Metal-InsulatorMetal) used in our experiment is shown in Figure 4. Two metal conductors are attached to the skin 5 centimeters apart. Opposite charges are then provided on the two conductors to create a bias condition, which interacts with electric dipoles under the skin, resulting in measurable capacitance values. The principal factors affecting skin capacitance measurements are autonomic nerve system (ANS) and central nerve system (CNS), and signals from these systems may induce changes in distribution and status of the electric dipole in the inner skin through charge transfer.

The aforementioned explanations indicate that skin signals can be effectively measured using electrical capacitance. This study included the use of skin capacitance measurements as a basis to determine the differences between signals in Stages $\mathrm{W}$ and dozing and the design of a device that is based on measuring skin capacitance and can be used for personal identification. Although these novel approaches are both interesting and challenging, they have not been discussed in the literature. In this study, investigations were conducted on these subtopics to uncover the unique characteristics of skin signals. The following discussions facilitate the understanding of the origin and mechanisms of skin signals.

\subsection{The Skin Signal Model and the Response Mechanism.}

Because the skin is similar to a large wrap covering a person's body, it plays multiple roles and serves various functions, the four most essential of which are (1) to protect the body's internal organs; (2) to flush waste (in the form of heat and body salts) and sweat out of the body; (3) to regulate the body's internal and external organs; and (4) to collect physiological information.

Concerning the collection of physiological information, the skin is assumed to be the channel enabling bilateral communication between the skin and the body organs as well as the nerve system, through which body signals are sent and received. Because the human body is governed by two nerve systems, the CNS and the ANS, the extent to which the two systems intervene with body functions varies for different consciousness levels. The CNS operates when an individual is awake and the ANS operates when she is asleep. When individuals are snoozing or in a light sleep state, the two nerve systems "fight" to gain control of the body, which generates a cyclic and complex control alternation process. Therefore, this study used the capacitance of the skin for measuring such changes.

\subsection{The Basic Skin Signal Capacitance Measurement Results.} This study was initiated by measuring skin signal capacitance, which can be used to provide reasonable explanations for future studies or other applications for which obtaining complex measurement data is required.

The time domain analysis results are shown in Figure 5. The subjects sat in a static position when the capacitance measurement was performed between Stages W and dozing under no bias. The duration of the data collection was 2 minutes, and the measurements were conducted once every 0.05 second. In Figure 5(a), the periodical changes in the data indicate that skin signals exhibited relatively constant fluctuation when no external stimulation was administered. Because the measurements were conducted between Stages $\mathrm{W}$ and dozing, differences in the data trend of the skin capacitance measurements were minor and not relevant. Thus they will not be further discussed.

Figure 5(b) shows the data for the subjects measured with a bias voltage of 5 volts, between Stages W and dozing. Measuring duration and period were the same as before. The signal characteristics change in the measurement data indicated that when bias was administered, considerable differences between the skin signals produced at Stages $\mathrm{W}$ and dozing occurred. This result suggests that when struggling against sleepiness, the physiological environment of the human body exhibits characteristics that differ from that exhibited when people are in a calm mental state. Physiological signals reflect physical status and emotional expression, which are crucial factors that influence socialization and facilitate the development of emotional understanding. Common methods of expressing emotions include facial expressions, gestures, tone of speech, word choice, posture, and physical reactions. Changes in physiological signals can also be used to observe emotional alterations.

Research on the electrical activity of the skin was first conducted by the French neurologist Jean Charcot in the late nineteenth century. The English physiologist Vigourous used the skin resistance of patients as a clinical diagnostic tool. Fere, who was working in the same lab as Charcot, determined that resistance could be decreased temporarily when administering a current between two electrodes attached to the skin. Subsequently, The Russian psychologist Tarchanoff attested that changes in the electrical potential between two electrodes attached to the skin could be measured without administering a current. Our experiment is unique in that we measure capacitance without providing any current through human body and Figure 5(b) shows that we were able to detect dozing stage instantaneously. 


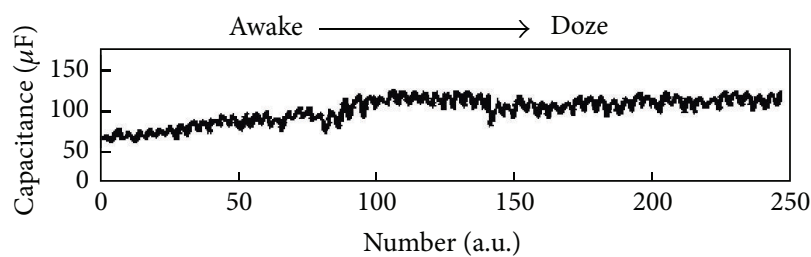

(a)

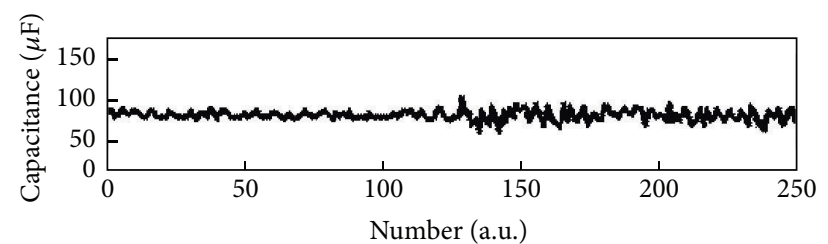

(b)

FIGURE 5: Five-minute capacitance measurement data obtained between Stages W and dozing with (a) no bias and (b) bias $=5 \mathrm{~V}$.

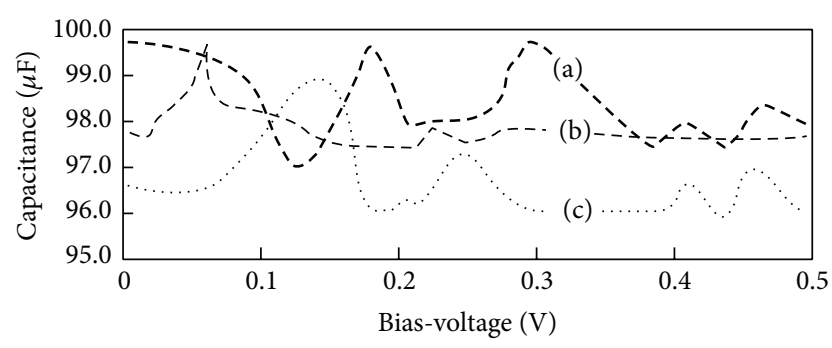

Figure 6: Administering bias between 0 and $0.5 \mathrm{~V}$ for $C-V$ scanning. (a) to (c), respectively, represent three of the subjects.

Figure 6 shows three of the 20 subjects who were tested during Stage $\mathrm{W}$ using a nested probe with small biases between 0 and $0.5 \mathrm{~V}$. Every measurement curve in the detection range of providing bias was repeated and can be used for identification. In future smart applications, providing bias skin capacitance measurements can be used for access control or identification systems because each individual possesses a unique capacitance curve.

\subsection{Differences in Skin Signals When Awake and When Asleep.} The two charts in Figure 7 summarize experiment measures with bias voltage and average capacitance as $x$ - and $y$-axes. Figures $7(\mathrm{a})$ and $7(\mathrm{~b})$, respectively, show the capacitance measurements obtained at Stages W and 1 using a nested probe with a bias between 0 and $5 \mathrm{~V}$. The amplitude of the measurement curves was mild during Stage W, while the amplitude at Stage 1 differed considerably and exceeded that of Stage W by sixfold.

Figure 8 shows a magnification of the red boxes in Figures $7(\mathrm{a})$ and 7(b). By comparing Figures 7(a) and 7(b), critical changes in the amplitude of the capacitance curve were determined to occur when the providing bias $=3 \mathrm{~V}$. If the peak value surrounding the area with a $3-\mathrm{V}$ providing bias exceeded $150 \mu \mathrm{F}$, the subject entered a state of dozing. Dozing could also be identified when the maximal and minimal peak value of the curve exceeded $90 \mu \mathrm{F}$. A possible explanation for why the amplitude of the capacitance curve was more substantial during dozing is that the ANS released numerous intense signals to wake the body. These signals are a source of positive and negative waves, which can cause constant capacitance changes. These changes are then amplified by providing bias. The present study is the first to present these groundbreaking results, and we are in the process
TABLE 1: Subject demographics.

\begin{tabular}{lcc}
\hline Parameter & Mean \pm Std & Range \\
\hline Sex & 38 males and 2 female \\
Age (years) & $30 \pm 10.2$ & $20.2-41.1$ \\
Body mass index (BMI) $\left[\mathrm{kg} / \mathrm{M}^{2}\right]$ & $24.4 \pm 3.4$ & $20.2-31.2$ \\
Test data (at 3.0 V bias) & & \\
$\quad$ Awake $(\mu \mathrm{F})$ & $96.4 \pm 2.4$ & $80.2-105.2$ \\
$\quad$ Doze $(\mu \mathrm{F})$ & $94.4 \pm 8.4$ & $48.2-165.2$ \\
\hline
\end{tabular}

TABLE 2: Comparison of all signals of the individuals when awake and when dozing.

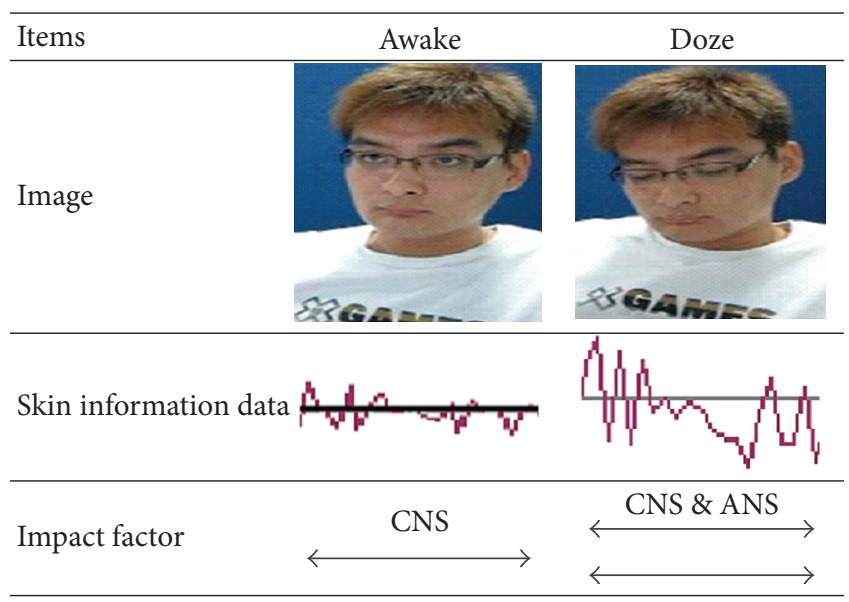

of designing more rigorous experiments for developing additional skin capacitance applications. The subject demographics are presented in Table 1.

Table 2 compares all the signals of the individuals when awake and when snoozing, clearly showing that the capacitance measurement method can be used to immediately indicate when individuals are begin to snooze, making the measurement method a sound discovery. When individuals snooze, the CNS and the ANS compete continuously to gain control of the body $[18,19]$, which causes the skin capacitance value to exhibit a cyclic, complex, and highly unstable control alternation process. Because the cyclic capacitance curve can be used to easily identify the two forces of influence controlling the snoozing process, the study's research team 


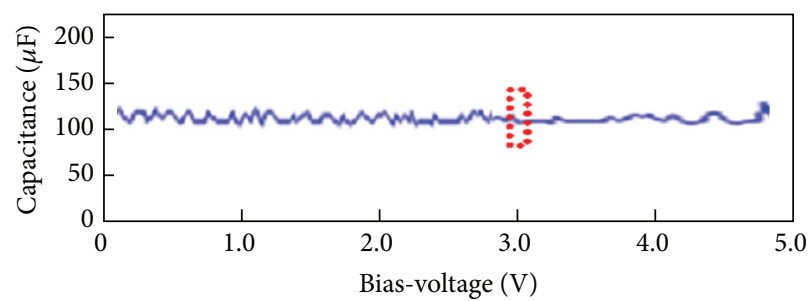

(a) Awake

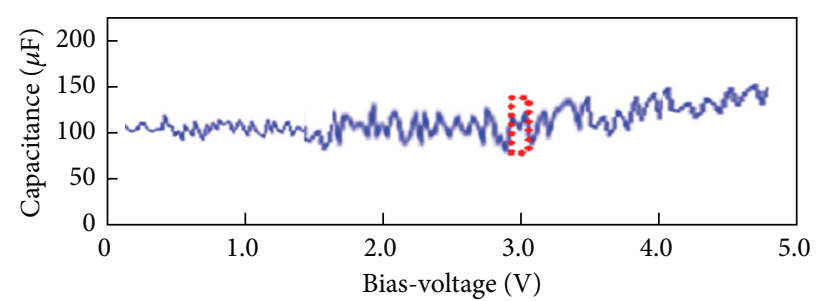

(b) Doze

FIGURE 7: Administering bias between 0 and $0.5 \mathrm{~V}$ for $\mathrm{C}-\mathrm{V}$ scanning at Stages $\mathrm{W}$ and dozing.

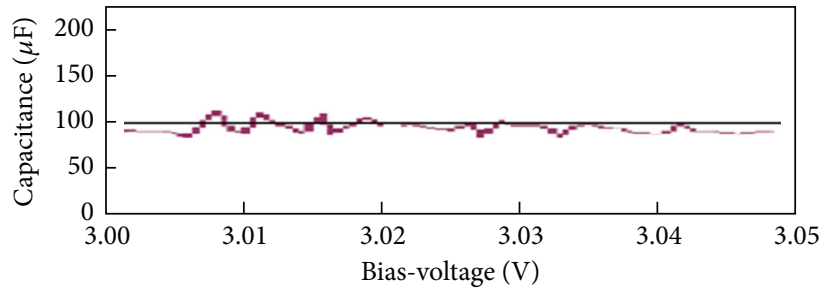

(a) Awake

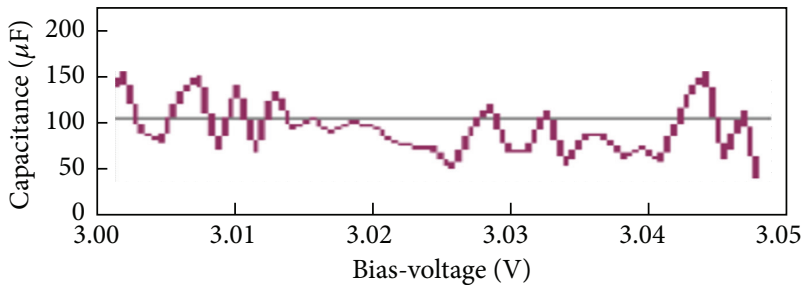

(b) Doze

FIGURE 8: An amplification of the red boxes in Figure 7.

is using the deconvolution technique for determining the roles that the CNS and ANS play when individuals snooze. The results of this study will be published in the next paper.

\section{Conclusions}

The present study proposes using capacitance as an alternative for skin impedance and conductance measurements to investigate the advantages provided by skin signals. The results indicated that analyzing capacitance using a (1) nested probe and (2) capacitance measurements to measure physiological and psychological signals is the optimal method for intercepting skin signals. The study design was developed based on the fact that the thin stratum corneum is an ideal insulator that intensifies the signal characteristics of capacitance changes. The experimental results confirmed that, by applying providing bias as a mild stimulation, capacitance curves can be used for developing future smart applications, such as access control and identification systems.

Additionally, when the subjects were given a bias between 0 and $5 \mathrm{~V}$ during the dynamic change scan and capacitance measurements, the amplitude during dozing differed considerably and exceeded that of Stage W by sixfold. Therefore, this measurement method can be successfully developed for extracting the characteristic signals of dozing.

The results of the present study are crucial for developing future applications of smart and remote medical systems. In future studies, the researchers will identify additional advantages and application methods involving skin signals.

\section{Conflict of Interests}

The authors declare that there is no conflict of interests regarding the publication of this paper.

\section{Acknowledgments}

The authors would like to thank the Ministry of Science and Technology and Ministry of Education of the Republic of China, Taiwan, for financially supporting this research under Contract nos. NSC 101-2221-E-214-032 and NCUT-14-E-CL039, respectively. Professors S. H. Chen and P. L. Lee are also appreciated for valuable discussions. Taiwan Advanced Semiconductor Engineering Package Inc. (TASEP) is also commended for allowing us to use their facilities.

\section{References}

[1] D. R. Bach and K. J. Friston, "No evidence for a negative prediction error signal in peripheral indicators of sympathetic arousal," NeuroImage, vol. 59, no. 2, pp. 883-884, 2012.

[2] D. R. Bach, J. Daunizeau, K. J. Friston, and R. J. Dolan, "Dynamic causal modelling of anticipatory skin conductance responses," Biological Psychology, vol. 85, no. 1, pp. 163-170, 2010.

[3] W. Boucsein, Electrodermal Activity, Springer, New York, NY, USA, 1992.

[4] V. I. Spoormaker, K. C. Andrade, M. S. Schröter et al., "The neural correlates of negative prediction error signaling in human fear conditioning," NeuroImage, vol. 54, no. 3, pp. 2250 2256, 2011.

[5] A. Bechara, H. Damasio, D. Tranel, and A. R. Damasio, "The iowa gambling task and the somatic marker hypothesis: some questions and answers," Trends in Cognitive Sciences, vol. 9, no. 4, pp. 159-162, 2005.

[6] Y. Shiihara, T. Nogami, M. Chigira et al., "Sleep-wake rhythm during stay in an intensive care unit: a week's long-term recording of skin potentials," Psychiatry and Clinical Neurosciences, vol. 55, no. 3, pp. 279-280, 2001. 
[7] Ö. Özkan, M. Yildiz, S. Bilgin, and E. Köklükaya, "Measuring the sympathetic skin response on body and using as diagnosispurposed for lung cancer patients by artificial neural networks," Journal of Medical Systems, vol. 34, no. 3, pp. 407-412, 2010.

[8] D. T. Lykken and P. H. Venables, "Direct measurement of skin conductance: a proposal for standardization," Psychophysiology, vol. 8, no. 5, pp. 656-672, 1971.

[9] A. Bechara, D. Tranel, H. Damasio, and A. R. Damasio, "Failure to respond autonomically to anticipated future outcomes following damage to prefrontal cortex," Cerebral Cortex, vol. 6, no. 2, pp. 215-225, 1996.

[10] M. A. Burock, R. L. Buckner, M. G. Woldorff, B. R. Rosen, and A. M. Dale, "Randomized event-related experimental designs allow for extremely rapid presentation rates using functional MRI," NeuroReport, vol. 9, no. 16, pp. 3735-3739, 1998.

[11] J. T. Cacioppo, G. G. Berntson, J. T. Larsen, K. M. Poehlmann, and T. A. Ito, "The psychophysiology of emotion," in The Handbook of Emotion, M. Lewis and J. M. Haviland-Jones, Eds., pp. 173-191, Guilford Press, New York, NY, USA, 2nd edition, 2002.

[12] H. D. Critchley, "Electrodermal responses: what happens in the brain," Neuroscientist, vol. 8, no. 2, pp. 132-142, 2002.

[13] P. M. Jenkinson, S. R. Baker, N. M. J. Edelstyn, and S. J. Ellis, "Does autonomic arousal distinguish good and bad decisions? Healthy individuals' the Iowa gambling task," Journal of Psychophysiology, vol. 22, no. 3, pp. 141-149, 2008.

[14] Y. Nagai, H. D. Critchley, E. Featherstone, M. R. Trimble, and R. J. Dolan, "Activity in ventromedial prefrontal cortex covaries with sympathetic skin conductance level: a physiological account of a "default mode" of brain function," NeuroImage, vol. 22, no. 1, pp. 243-251, 2004.

[15] E. U. Weber and E. J. Johnson, "Mindful judgment and decision making," Annual Review of Psychology, vol. 60, pp. 53-85, 2009.

[16] A. A. Reid and C. González-Vallejo, "Emotion as a tradeable quantity," Journal of Behavioral Decision Making, vol. 22, no. 1, pp. 62-90, 2009.

[17] S. Mookerjea, R. Krishnan, S. Datta, and V. Narayanan, "Effective capacitance and drive current for tunnel FET (TFET) CV/I estimation," IEEE Transactions on Electron Devices, vol. 56, no. 9, pp. 2092-2098, 2009.

[18] X. Long, P. Fonseca, J. Foussier, R. Haakma, and R. M. Aarts, "Sleep and wake classification with actigraphy and respiratory effort using dynamic warping," IEEE Journal of Biomedical and Health Informatics, vol. 18, no. 4, pp. 1272-1284, 2014.

[19] J.-M. Lee, D.-J. Kim, I.-Y. Kim, K.-S. Park, and S. I. Kim, "Detrended fluctuation analysis of EEG in sleep apnea using MIT/BIH polysomnography data," Computers in Biology and Medicine, vol. 32, no. 1, pp. 37-47, 2002. 

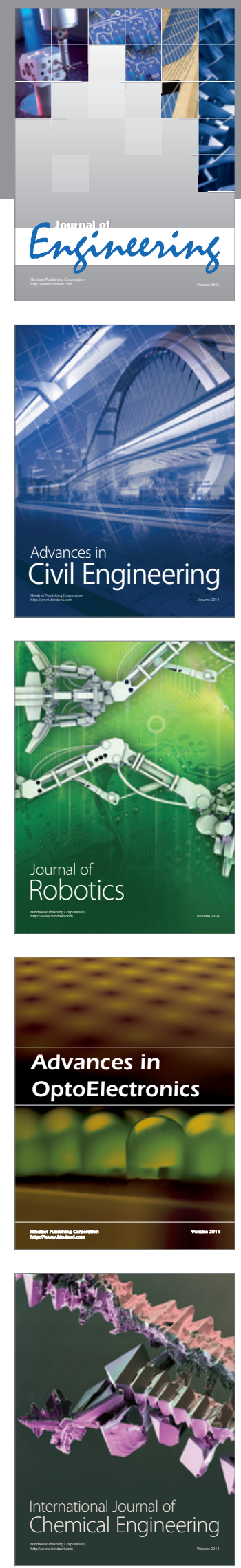

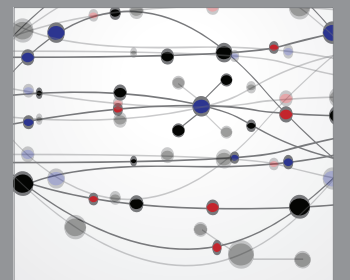

The Scientific World Journal
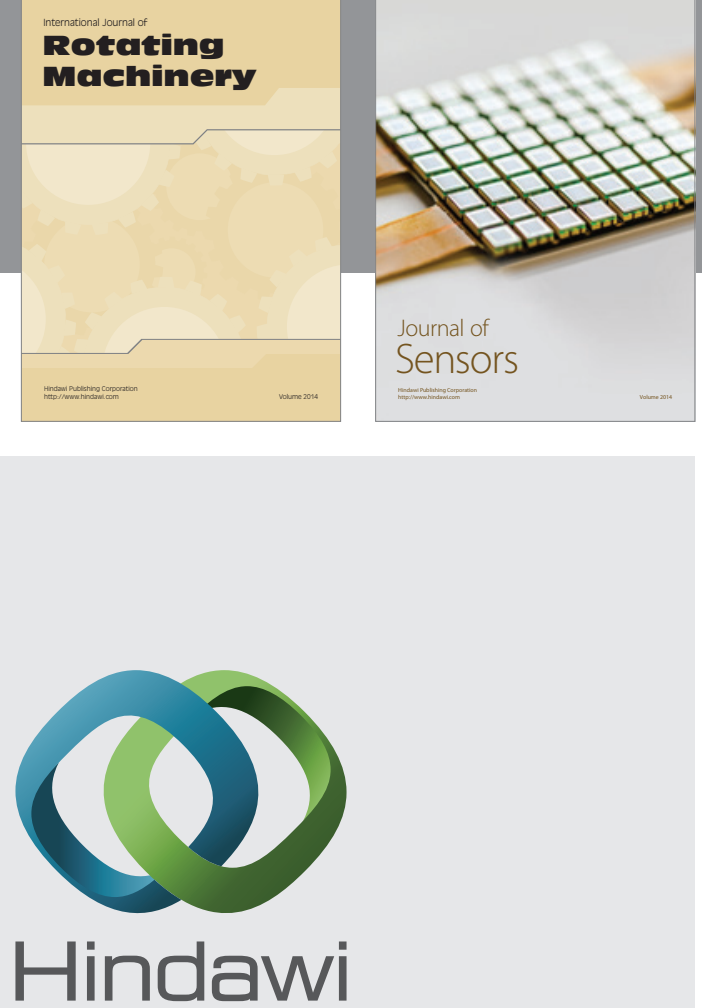

Submit your manuscripts at http://www.hindawi.com
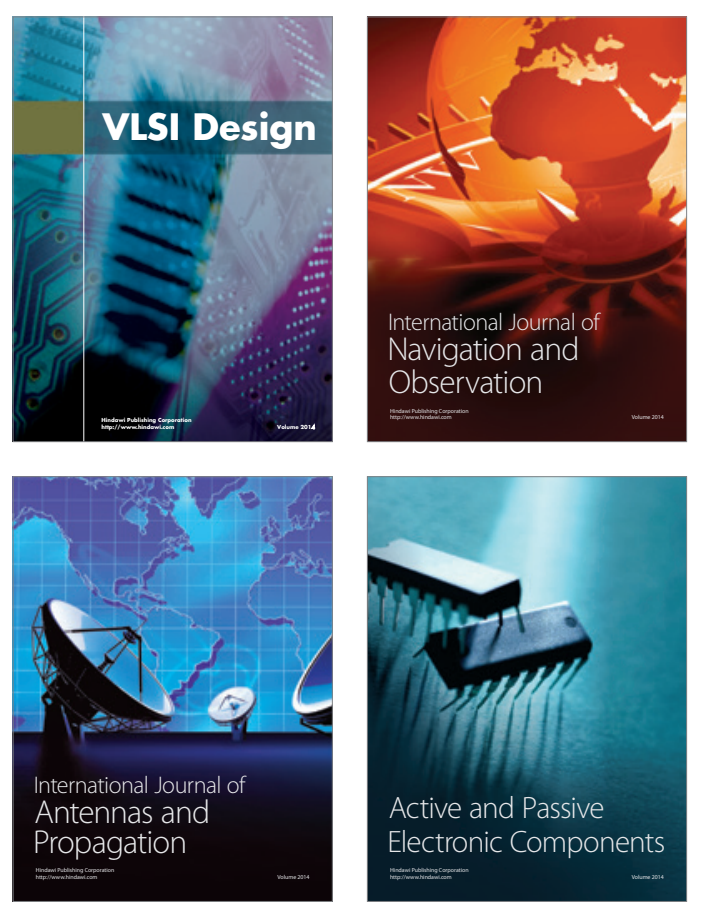
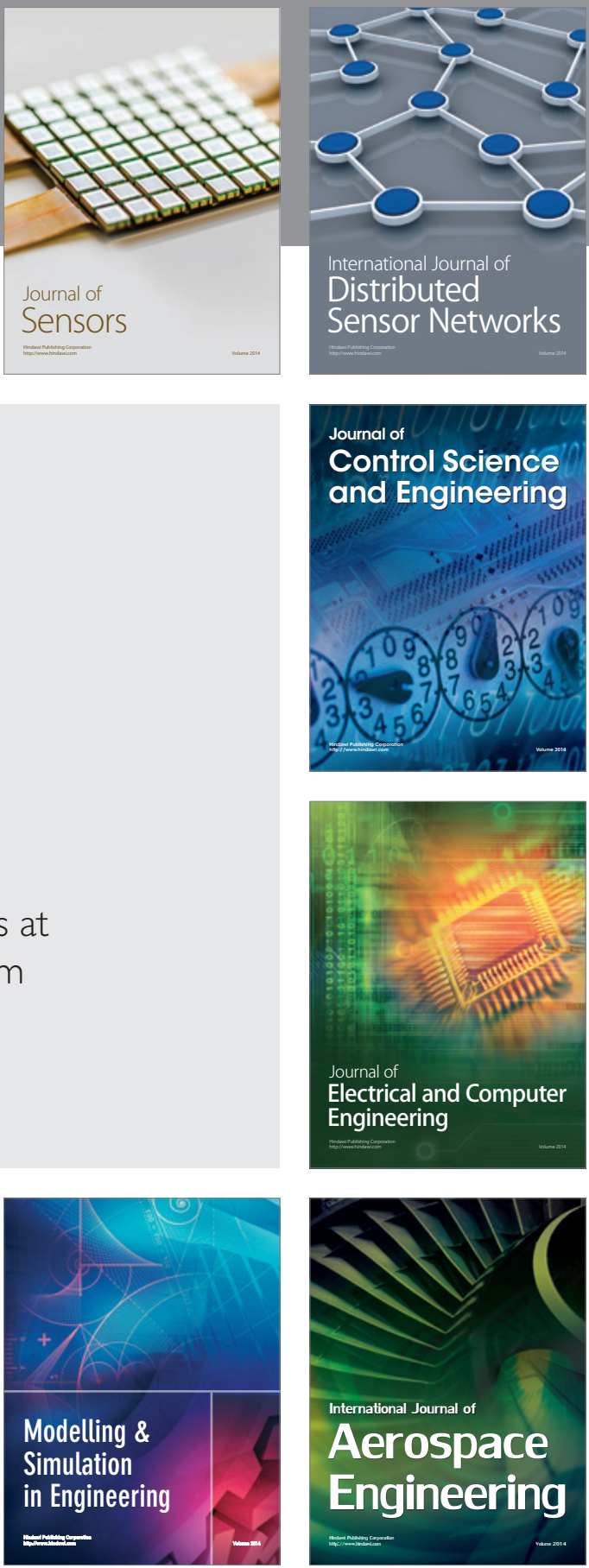

Journal of

Control Science

and Engineering
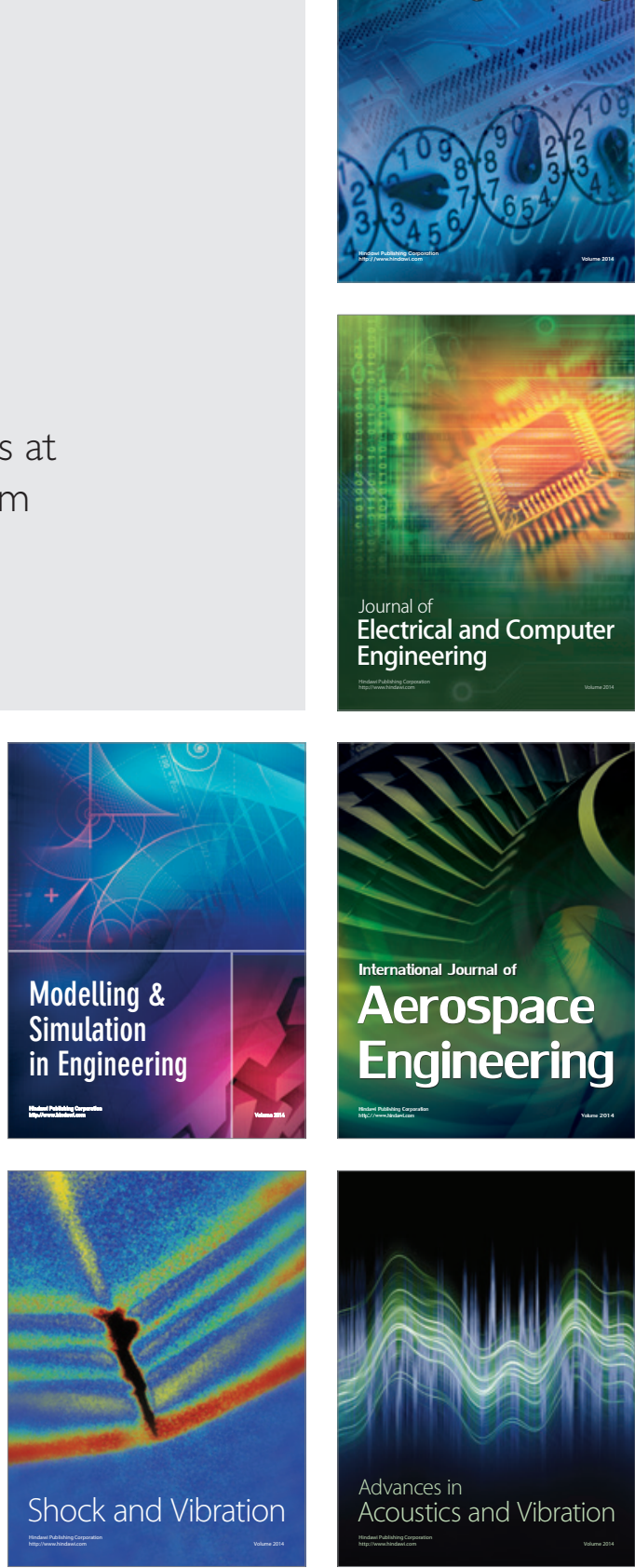